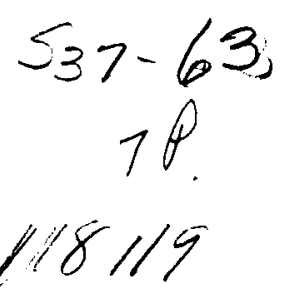

\title{
A Framework for Qualitative Reasoning About Solid Objects
}

\author{
E. Davis \\ New York University \\ New York, NY 10012
}

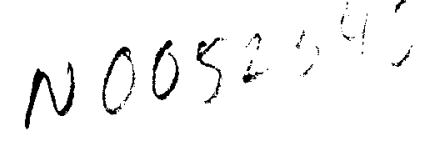

\section{Abstract}

Predicting the behavior of a qualitatively described system of solid objects requires a combination of geometrical, temporal. and physical reasoning. Methods based upon formulating and solving differential equations are not adequate for robust prediction. since the behavior of a svstem over extended time may be much simpler than its behavior over loeal time. This-paper discusses a firstorder logic. in which one can state simple phivsical problems and derive their solution deductivelv, without recourse to solving the differential equations. This logic is substantially more expressive and poweriul than any previous $\mathrm{Al}$ representational system in this domain.

\section{Introduction}

To operate effectively in an uncontrolled environment, an autonomous robot will have to reason about. understand. and predict external physical events. In many circumstances. however, it will be necessary to reason about physical events on the basis of partial information: the objects involved may not be wholly perceived. or the complete physical specifications may be too complex to use, or the rotot may need to reason about hypothetical or generic situations. In such cases. the robot will have to reason qualitatively, inferring general characteristics from incomplete knowledge. Human common sense is often very good at speedy prediction of physical events in qualitative terms: conventional computational schemes are typically very poor at it.

Lnderstanding solid objects is particularly important in physical reasoning. and human beings are particularly adept at thinking about solid objects. Our objective is to build an AI program that in reason quaditatively about solid objects and that an derive comect predictions about their behavior in ases where these predictions are innuitively obvious. This is harder than one might itrt guess, owing to the many complex ways in which the zeometry of the cbjects affects their behavior.

As a first step toward building sucin a program. we have analyzed the kinds of knowledge needed to support such reasoning. and we have defined a formal language $L$ in thich this cormaj arguage an te expressed. Tie inve snown that interesting problems can be solved qualitatively hy inference from plausible axioms expressed in $L$. The languige $L$ is more expressive and supports richer inferences than 1 yy previous representation scheme in this domain. We zive the iull details of $L$ and its applications in [i]: here. we give only a sketch.

In oncentrating on the representation and formulation of knowledge. and postponing questions of algorithms or control structure. we follow Haves [21. Houcver. we depart from Haves research program in some respects. Hie do not aitempt to model nave" phrsics: rather. we have made free use of Newtonian mechanics, including concepts that have no commonsense analogue. such as total mechanical energy. Also, our proots are lengthy, violating Hayes' dictum that obvious facts should have sinort proots.

The mathematics used here is not "qualitative" in the restricted sense of representing quantities purely in terms of order relationships and constants [3]. Such a representation is too weak to support the inferences needed in this domain.

We have chosen two kinds of problems as foci for our analysis; precticting what happens when a die is dropped inside a funnel (Figure 1) and what happens when a block is dropped onto a table (Figure ?).

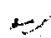

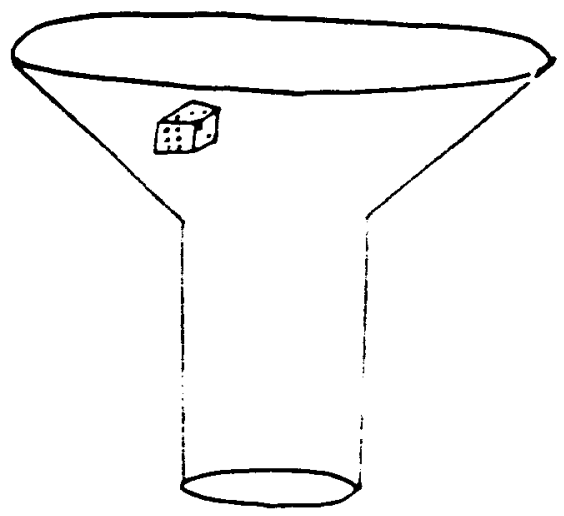

Figure !: A dic is released inside a funnel

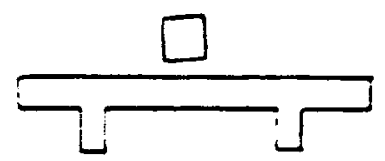

Figure :: A block is released onto a tacle.

Different fonns of these problems involve $a$ rich. interconnected body of geometric and physical knowledge for their solution. This paper will focus primarily on the "die in the funnel" example.

\section{Baxkground}

Several previous AI projects have studied the qualitative phisics of solid objects. For example. Fahiman's BL7LL program [4] determined the stability of a tower of polynedral blocks. De Klects NEWTCN [5] prediced the hethavior of a point mass sliding on 3 
constrain:- Bundy's MECHO [6] used force analysis and conservation lawrs to make physical prectictions in situations of specialized format. Forbus' FROB [7] predicted the behavior of a point mass flying among constraints. Funt's WHISPER [8] predicted the behavior of a collection of objects by simulating it in a retina-like image. Novak's ISAAC If] identified English-language programs of fixed form, and applied special case equations to them. Shoham [10] analyzed the local mobility of an object within constraints.

All these programs provided valuable insights. They were, however, limited in geometrical expressivity and in the range of physics understood. Of these systems, only BUILD dealt with three dimensions; and onily MECHO dealt with the motion of extended objects. Oniy a few kinds of physical interactions were considered.

Another limitation of these programs was more subtle, but more fundamental; they were based almost entirely on extrapolating differential behavior. To make a prediction, the program first determined how each state of the system will tend to change, and then extrapolated these changes to preatict a continual trend of change up to the point that the structure of the system changes. This extrapolation could be done qualitatively, as in FROB and NEWTON, or symbolically, as in MECHO, or using point-by-point simulation, as in WHISPER, or by numerical integration, as proposed by McDermott and Bermecky (personal communication).

For example. FROB [7] predicts the behavior of a bouncing ball in a well by dividing physical space into + regions the interior of the well, the bottom, and the two sides), and dividing the velocity space of the ball into nine (motionless, up, down, left, right, and the four guadrants.) (Figure 3) There are thus 27 possible states of the system. ( $4 \times 9.9$ impossible states). The laws of physics are then used to determine which iransitions between states are allowed. and thus a transition graph of states is developed. FROB predicts that the system follows a path in this transition graph. ending in a stable state of rest.

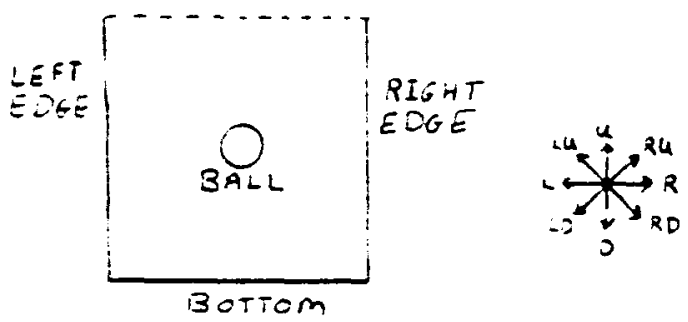

Figure 3: Discretized space and velocity in FROB

However extrapolation is fone, simulation is inadecuate for robust prediction. In this kind of anajysis. each different set of boundary conditions is a different ivstem state Each iuch itate rust te ieparately tetected categonzed, and analvzed. and the svstem is progress through these states must be recorded. Often, iowever. such 3 eategorization is difficsit and poirtless. Consider the problem in figure 1: 3 small die is released inside 3 large steep funnel. Many states are possible: the die may be in free-fall: it mav be coiliding or in continuous contact with the top or bottom pan of the funnel ivennet on any of eight vertices. twetve sides, or six faces: it nav be spinning. sliding, or rolling. up. down, or around the funnel. But the prediction that the die comes out the funnel does not iequire the enumeration of the states and the paths through them.
There are two further arguments. First, the sequence of states traversed depends delicately on the exact shapes. sizes, and physial properties of the die and the funnel. while the conclusion that the die comes out the bottom is robust with respect to small variations in these parameters. Therefore, if the problem is specified with some small degree of imprecision, simulation will either be impossibte. or involve some monstrously branching tree of possibilities. But in qualitative reascning the prediction that the die comes out the bottom should be almosi as easy with imprecise data as with precise. Second, the complexity of simulation goes up rapidly with the number of interacting objects. In figure 4, for example, with one die inside another dropped inside a funnel, the set of system states is the cross product of the possible interactions of the two dice with the possible interactions of the outer die and the funnel. Nonetheless. the prediction that the two dice will come out the bottom is intuitively almost as easy as with. only one die.

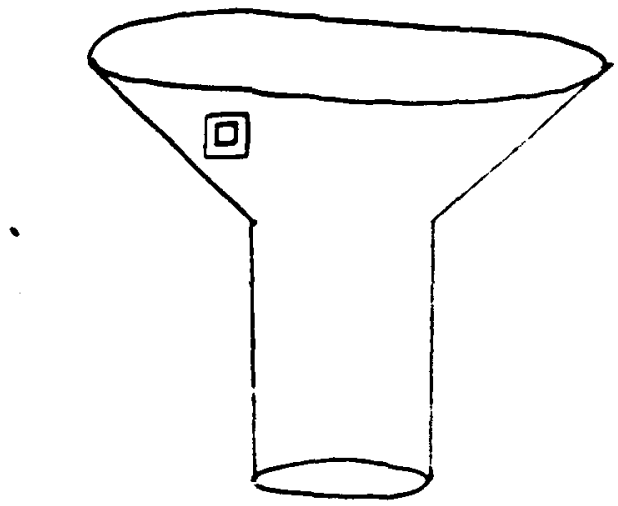

Figure 4: One die inside another released inside a tunnel

In short, formulating and solving jifferential equations is an inadequate technique in this domain. since the behavior of these physical systems over ex.ended time is often essier to sharacterize than their behusior over local time. A poweriul physical reasoning iystem must be able to infer the general quality of a course of events from broad characterizations of the physial properties of the objects involved, without salculating excin subevent.

The programs sited do use some techniques besides simulation. MECHO and NEWTON use energy conservation to prune possible svstert behaviors. Any state with more nechanical energy than the starting state can be ruled out is 1 possibility in all tuture states; for example the die cannot come out the top of the funnel. FROB predicts that the system ends in a stable state. We believe that effective qualitative reasoning requres more inferences like these, and less use of simuiation.

A natural krowledge engineering approach would use rules that state the desired prediction ivis is it simall object released inside 1 steep. large-mouthed funnel will fall out the bottom. "But rules of this kind are inadequate, and have rightly been rejected by previous researciters. Any single such rule covers only 1 imall dass of problems: covenng large classes of problems requires many separate disconnected rules. In particular. 1 rule like the one suggested above applies oniv when the tie and the funnel are the only objects involved. As soon as another object enters, the rule gives no guidance. That is. such rules are not compositional zeross ubjects. Even without other objects. if we allow wide tanges in the siape of the 
die and the funnel, the conclusion ${ }^{-}$will sometimes apply and sometimes not. Since there is no simple, general nule for when the die comes out the bottom, a different rule must be stated for each special geometric case.

Maintaining a knowledge base with many special case rules is not effective. First, the knowledge base will have to be large and inefficient. Second, if a new case is not precisely covered in pre-canned categories, the system cannot even begin to deal with it. Third, this approach is aesthetically distasteful. A well-designed system should use similarities among different cases of a die falling through a funnel, and similarities between this problem and similar probtems. such as a die shot through a tube. or a die dropped into a box. The analyses of these cases ought to have more in common than the use of rules which are syntactically similar. Finally, it seems plausible that an integrated system of rules will support learning better than a tabulation of special cases.

\section{Examples and Analysis}

We propose that the "die in the funnel" can be analyzed as foliows: (i) Due to the topology of the funnel. if the die yoes from inside it to outside it. the die must either exit the top or exit the bottom. (ii) Since the die is tropped from rest inside the funnel, it cannot have the energy to exi de top of the funne. (iii) There is no stable resting point for the die inside the funnel, since it is smaller than the funnel's mouth. and the funnel's sides are steep. (iv) The die annot stav corever moving within the funnel. For its kinetic energy will eventuailv be dissipated. Therefore. the die must axit the bottom of the funnel. We claim that in most ases where common sense predicts that the die will some out the bottorn, it wiil be possible to carry out suct an anaivsis. and to support the substeps by inferences irom gencral rules. Different problems wi!l vary in the usilfications of the substeps.

Related problems will share parts of the analvsis. For instance. in predicting that $d$ die in a small-necked funnel will come to rest it he :op of the neck. we mav use the identical arguments (i) :nat the die must either exit the top. exit the bottom. or stav inside; (ii) that it sannot exit :he top; and (iv) that it annot itav inside in a xerpetwal state of motion. The argument $(11 i)$ that it :annot rest stably inside the iunnel rust be nodifted to an argument that it can only iest staoly at the top of the ne-x of ine iunnel: and :he auditional arjument must be made that it annot exit the bottom of the tunnel. since the urifice is to smail.

This analysis avoids both problems discussed in section ?. We can avoid inaivsing, or even determuning, the states of motion of the die inside the iunnel: jil tie need to determine is that the die cannot rest stably inside. Different ategories of problems are analvsed in simiar but not identioal ways from general principies.

In the iest of this section. we look 3 t variations $x$ this exampie, and snow how this analysis an be iopied.

We begin with a simple ase ifigure 5 . The die is 3 uniform sphere. The iunnel is the iurface ji evoiution about a vertical $3 x$ is of a pianar if side. The ractius of the Jie is iess than the radius of revolution of the funnel. The steps of the argament are

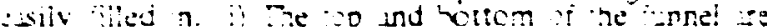
the univ unitices of free soace connecting the inside of ine innnel with its outside. Therefore. if the tie is to go trom inside to uutside. it must go through the top or ibe bottom. (ii) Since the die is spherical, its zenter of mass is in its interior. Since the :op of the iunnel is horizontal. und directed upward, if the die were :o exit it. sach point in the interior of the die would be above the top of the iunnel at some ime. In particular. the senter of mass would be above the top it some time. But the die started vut irom rest below the top of the funnel. and there is no source of udditional energy for the die. Theretore. tine tie annot come out ihe tor. (iii) By a geometncal argunent. the die can only abut the inside of the funnel in a single point. A uniform sphere can be stably supported at a single point only if the supporting surface is horreontal there. The inner surface of the funnel is nowhere horizontal. Hence there is no resting place for the die inside the funnel.

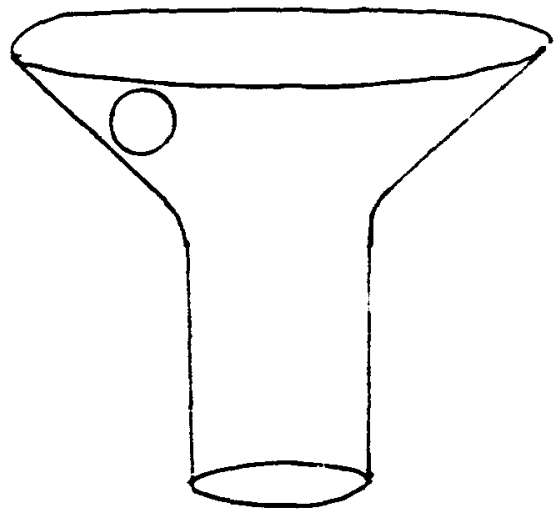

Figure 5: A spberial die inside a radially symmetric innel

\subsection{Out the top, out the bottc $m$, or stay inside}

We now consider how this argument an be generalized and modified. (Further modiñcations are discussed in [1].) Part (i), that th: die must either zit the top, exit the bottom. or stay insid. Joes not require that the funnel be a solid of revolution; it requires oniv that the funnel be a tube with only two onfices. weaken the condition further, and require only that all orifices other than the top or the bottom be :00 snalal to let the die through. Determining whether 1 die an go through a hole is ar. easy geometric calculation ior : arious special cases.

\subsection{Yot out the top}

Part (ii), the argument irom energy conservatco that the die cannot come out the top. depends on tie die reing convex and on the center of mass if the die starting out below any part of the iop. Convexity is inly id to establisn that the center of mass of the voject is in its interior. If this can be done otherwise - for exarrie. by exact alculation, or 'ov establishing that the joiec shape is a small perturbation of a convex shape. - בat is suificient.

A still weaker ifficient condition is that the rnged illing in of the die is convex. The anged tilling a oi a three-dimensional shape $\sigma$ is jefined is follows: Cinsider inv pianar ctoss section of $S$. Let $C$ be any impic sosed

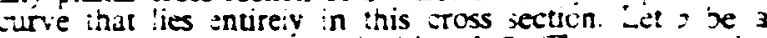
coint in the riane $n$ ine inside of $C$. Then 2 is the inged illing in of $S$. Figure o)

Lat $S$ be :he shape of some $0^{r}$ eat $O$. y.d let $R$ re the inged illing in of $S$. Assume $R$ is convex. Cearr. $R$ is

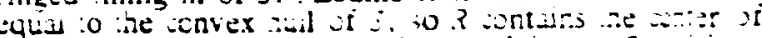
nass of $O$. Let $C$ be 1 dosed eurve iving in $S$ ino in 3 plane containing the zenter of nass of 0 . If $E$ zoes through I planar suriace. then so joes every point nside $C$. Thus, if $O$ exits the top of the funnel, isen ise enter oi mass of $O$ must ikewise. and the proot zoes itrough Thus we in stablisi step (ii) for such shapes is a :orus, 3 wiffle ball. or a cratered convex sinap:

\subsection{Vo resting point inside}

Part (iii). ihe argunent that the jie vannot iest :nside the tunnel. depended in our iirst sxample on the itrong 


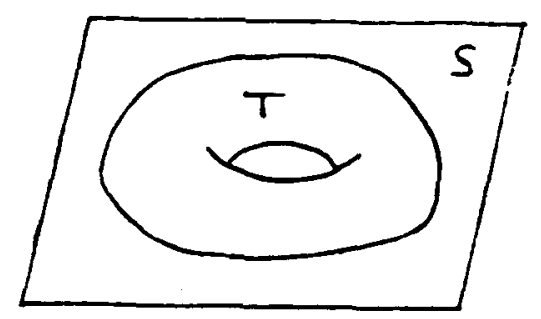

Torus $\mathrm{T}$ is cut by plane $\mathrm{S}$

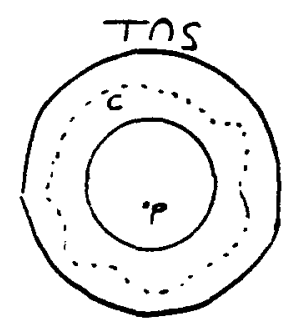

Cross section of $T$ by $S$

$C$ is a curve in the cross section. $P$ is a point inside $C$.

Figure 6

assertions that the die was $a$ uniform sphere and that it could contact the funnel only in a single point. We can easily generalize to nearly uniform, nearlv spherical dice. The following formula holds: let $\theta$ be the siope of the support: let $\mu$ be the coefficient of friction: let $\phi$ be the maximum angle between the line fro:n the center of mass to a point on the surface and the normal to the surface at that point (Figure 7). The ball an stand still onjy if $\mu \geq \tan (\theta)$ and $\theta>\theta$. Similarly. if one die is a spherical shell containing another die, they rest stably only if the joint center of mass of the two dice is located directly above the contact point of the outer die with its suppori. and the inner die rests stably inside the outer die.

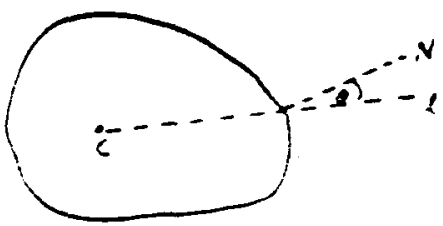

$\mathrm{V}$ is the nom:al to the surtice.

$z$ is the center of mass.

$i$ is a line through $c$.

$\rightarrow$ is the ingle between $N$ and $\mathrm{l}$.

Figure ${ }^{-}$: Dis:or:ed chere

If the die an contact the funnel in severat points with different suriace normals. the inalvsis becomis harder. The wider the range of the horizontal component of the surface normals at contact points, the stecper the slope must be, for the normal forces at the various contact points will tend to act aginst each other. Ind thus generate larger iriction forces. The following rule holds: Let $A$ be in contact with $B$. Let it be the minimum slope of the surisce of $B$ at 1 contace point. Consider the horizontal compunents of the iuriace normals of $B$ at the contact points, and assume that there is some direction which lies within some small angle $\phi$ of all these horizontal components. Let the coefficient of friction be $\mu$. If $\mu<\cos \phi \tan \theta$, then $A$ will slide down $B$.

Combining all the different ways in which the restes (i), (ii), and (iii) may be established, and all the wass in which their geometrical preconditions may be satisīed. gives a rich. interconnected body of results, all with the conclusion, The die falls out the bottom of the funne.

\section{The Block on the Table}

The behavior of the block on the table can be analyzed using a similar argument. After the bloci is released, it wil fall to the table, tipple over a bit, ind then move along the table in some combination of slicing. bouncing, and rolling. (Figure 8). It can be estimated bow long it will take for the friction involved in sliding and the inelasticity involved in bouncing to consume all the energy gained in the fall and the tipple, and how far the bloct can travel during that time. A similar estimation be made for roiling, as long as the object rolls sufficiently poorly. If the surface of the table is uniform. and if tese motions will not bring the block off the edge of the table, then it can be predicted that the block will attain a stable state of rest within the estimated time. and within the estimated distance of the point of release.

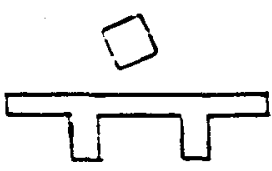

Block released.

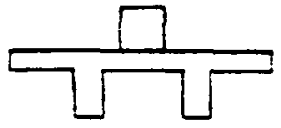

Block :ipples over.

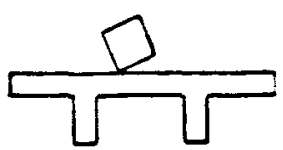

Block hits table.

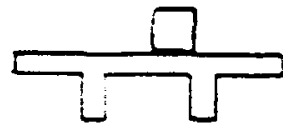

Block bounces

and slides to rest.
Figure 8: A block settling or a table

\section{The Underlying Knowledge}

\subsection{Geometry}

The arguments in section 3 used several diffent kinds of yeometric knowledge. in luding:

- The abilitv to name and describe particular point xets that ure connected to objects ind are liseful :or physical reasoning. such as the top and botiom $x i 3$ :unnel. These are called "pseudo-iojects" in sur svstem: the problem of constructing them effecueiv is the same as the problem of constructing beric tiagrams in FROB (9).

- Topological predicates. For example, the timel corms topologically a box with two orifices, and the ife starts out inside the box. [11]

- The use of a property quantified over il irregularities of 3 certain kind in an object. For instance. the funnel has no hoies iarge enough : $\supset$ 'et the die through other than the top and bottom.

- Special shapes, such as spheres und surfaces of rotation.

- Inequalities on metric dimensions. For example the iadius of the die is less than the radius of the furned. 
- The bounding of the range of the surface normal over a part of an object's surface. For example. we wish to say that the slope of the funnel is everywhere positive in its inner surface.

- Convexity and related properties.

Any adequate geometric language will be strong enough that these, or most of these, can be either expressed directly or inferred.

\subsection{Temporal Logic}

Our temporal logic follows McDermolt's [12]. A scene is an instantaneous snapshot of the universe. In our domain, a scene specifics the positions and velocities of all objects. A chronicle is a function from the time line to scenes. Chronicles include all continuous motion of objects through space, not just those that are physically possible.

The velocity of an object at an instant is defined to be the limit of its velocity from preceding time. Thus. we can speak of the velocity of an object at the instant of a collision.

The "frame" or "persistence" problem of determining what remains true over time $[12$. 13] does not arise. There are two classes of predicates in the domain. The first class includes predicates thai depend on position and velocity of objects. These are not issumed to remain constant over any interval unless proven to do so. The second class includes itructural predicates. depending only on the shapes and material properties of the ofjects. Ihese are always constant over the problem. and so are defined stemporalt. (The closed world assumption is made explicit through the predicate "isolated $\left(O O C^{\circ}\right)$ ", which asserts that, during $C$. no mohile object in the set of ohiects $O O$ ever comes into iontact with any object outside $O O$.)

\subsection{Physics}

The world consists of 1 inite set of iolid abiects moving in space through lime. Objects ure rigid and indestructible. The interior of shicts may not overlap. Objects have two internal properties hesides their thape: a distrioution of mass. and 1 coetficient of elasticity. which fetermines zow the obect tenaves in a collision. Any pair of ebiects have a coefifient of fiction. which determines the trictive corces between the obicets.

Objects are subject to four kinds of forces: 1 uniform Jownward gravitational force: normal forces. which act to prevent ubjects from vverlapping: iriction: and a weak urag force, which dissipates sinetic energy. Certain objects ure itred; ther do not move. whatever the forces

Vecessary physieal deductions include the collowing:

- Determining whe:her a ict of obiects ian ittain 3 stable scene while certain geometnc conditions told.

- Finding constrants on the iocation of the center of mass of an obiect or a set of vo ears.

- Resolvine 1 set of corces. Ind fetermining notion Incier inuse iorses.

- Predicting that the existing struture of contacts between ubjects will thange.

- Predicting a coilision.

- Predicting the result of a collision.

- Determining anether a saronicle riolates a conservation law

- Characterizing the faths that un obiert can take without coming is aserlap other ubiects

\section{Ontology}

The ontology for our language requires a number of sorts of individuals.

Quanrities. Instants of time. quantities of mass. quantities of energy. These are modelled as real numbers. $R^{3}$

Points and vectors. These are modelled as elements of

Point sets. Subscts of $R^{3}$.

Vector fields. These are functions from some point sets to the space of vectors. For example. the surface normals to an object in a fixed position, directed outward. form a vector field.

Rigid moppings. Mappings from $R^{3}$ to $R^{3}$ which preserve distunce and handedness. These specify a change in position.

General velocities. The derivative of a rigid mapping. A general selocity is the composition of 3 linear velocity and an angular velocity about a specified axis.

Objects. These are primitive entities. The shape of an object is the point set that it occupies in some particular standard position. This is assumed to be connected. closed, and normal.

Scenes. A scene is a snapshot of the world. Formally. it is a function which maps an object to 3 pair of a rigid mapping, giving the position of the object. and a general velocity. The place of an object in a scene is image of the object shape under the mapping associated with the objec in the scene.

Pseudo-pjects. These are point sats that "move around" with objects, like the hole of 1 doughnut, the opening of a bottle, or the center of mass of any ubjec. Formally, a pseudo-object is a pair of a cource object and a point set, designating the point set cocupied by the pseudo-object when the object is in standard position. $17 x$ place of a pseuco-object in a scene is the image of is shape under the ...apping issociated with its source obict in the scene.

Chronicles. A chronide is a function irom ar inter $\exists$ of time to scenes.

All chronicles are subiect to the following constraines:

i. All scenes in the range of the chronic:e tave the sare objects in their demain

ii. Cbiects nove continiously in space

iii. Obiea velocities are :ontinuous from gresious timex

iv. The velocity of an sbject is the ierivative of is pusition.

Chronicles do not have to be phrsicajly possible. We use the predicate "phys-poss $(C)$ " to distinguish chronicies that obey the laws of phistes.

\section{Axioms for Physical Reasoning}

Bascd on the above ontology. we save developed a iirst-order anguage $L$ and $a$ set of $2 x i c r s$ sciequate 0 solve the irst "Jie in the :unnel" example. The comple: undssis is rather !enethy: he language "ses thout nine: non-logical ierms. not induding the stariard arithme: operators. and the anadssis involves itwout $1+10$ exiors Vlost over : wo thirds; if the ierms ind axioms are

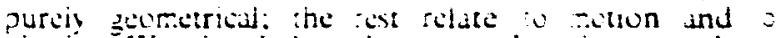
physics. "We give below inree iample ixisms. and we compicte statement of the "die in the tunnel example. $x$ illustrations.

Geon'etnc Axiom: Smoothness and the value of the surtace normal ure loced properties of the houndar: Specifically. if tho bodies share part of ineir houndar: then. at inv interior point of the overlar. ore is smovt? iff the other is smoith. Ind their iurize normals ase vither paralle! or anti-parallel. 
$[\operatorname{body}(X X 1) \cdot \operatorname{body}(X X 2) \cdot$

$X X A \subseteq$ boundary $(X X 1) \cap$ boundary $(X X 2)$.

$X \in$ interior $(X X A): \operatorname{smooth}(X X 1, X)\} \rightarrow$

( $\operatorname{smooth}(X X 2 X)$

$[\operatorname{surf-norm}(X X 1 X)=\operatorname{surf-norm}(X X 2 X)$

surf-norm $(X X 1, X)=-\operatorname{surf}$-norm $(X X 2 X) \mid 1$

Axiom of Motion: If an object $O$ has zero velocity in every scene of a chronicle $C$. then it stays in the same place throughout $C$.

$$
\begin{aligned}
& {[\forall, S i \operatorname{sonenes}(C) \text { \& } \operatorname{velocitv}(O S)=0] \text {, }} \\
& \forall \text { si.s: } S 1 \in \text { scenes }(C) \cdot S 2 E \text { scenes }(C) \text {, } \\
& \operatorname{mapping}(S 1, O)=\operatorname{mapping}(S 2 . O) \mid 1
\end{aligned}
$$

Physics axiom: The energy of an isolated set of objects $0 O$ never increases in a physically possible chronicle $C$.

[ phys-poss $(C) \cdot$ isolated $(O O, C) \cdot T 1<T 2]$,

energy $(O O$,scene $(C, T 1)) \geq \operatorname{energy}(C O$, scene $(C, T I))$

Problem statement: Consider a spherical die, and a radially symmetric funnel. Assume that the inner radius of the funnel is greater than the radius of the die: and that the inner side of a radial cross section of the funnel is convex. If the die is released inside the funnel, and the funnel is held fixed far from the uround, then the die will eventually fall out the bottom of the funnel.

\section{Constants of the example:}

odie - the die

otunnel - the runnel

$c$ - the chronicle

$x x$-piunnel - the planar form from which the tunnel is generated

$x x$-center-line - the axis of the funnel

$x$ center - a point on the axis of the funnel

\section{tssumptions:}

sphere( shape(odic))

(The die is 1 sphere.)

mobiler(exdie).

(The dit is not fixed.)

Shape(ofunnel) $=$

wlid-of-revolution(xx-prunnel.xx-ienter-line).

The tunnel is the solid of revolution of xx-ptunnel uround $\mathrm{xx}$-eenter-line.)

p'dnar(xx-piunnel I $x x$-center-line)

(x.x-plunnel is a radial sross section of the funnel.)

convex-sidet inner-dside(xx-piunnel.xx-cente-line). xx-piunnil).

(The inner houndary of $x x$-piunnel with respect to $\mathrm{xx} \cdot \mathrm{enter}$-line is convex.)

distance $(x x$-giunnel.$x x$-centerline! > radiuslodic) $>$ !) The radius of the funnel is greater than the radius of the die.)

rx-enterline = nake-inct renter., uo

(The axis of the funnel is vertical.)

standard-positioni ofunnel.startscene(c)).

(The iunnel is oriented in standard position.)

fixed(oiunnel).

(The iunnel is fixed.)

isolated (\{odie of unnel .oground\}.c)

(The die is isolated from even thing but the iunnel and the ground."
$X F \in$ shape (ofunnel) $\cdot X G \in$ shape(oground) ,

height $(X F)$ - height $(X G)>$ diameter(odie)

(The funnel is more than the diameter of the die above the ground.)

infinite(c).

(The chronicle is etemal.)

phys-poss(c).

The chronicle is physically possible.)

motionless(odie,startscenc(c)).

(The die starts from rest.)

place(odie,startscenc(c)) $\subset$

tube-inside( shape( of unnel), s-tube-top( shape( ofuncl)) s-tube-bot(shape(ofunnei)))

(The die starts from inside the funnel.)

Prove:

exits(odie,

pseudo-object (ofunnel,s-tube-bot(of unnel,sup)). c).

(The die exits the bottom of the funne!)

\section{Conclusions}

The strengths and limitations of this theory are evident. On the positive side: Using pure first-order logic, we give a formal snalvsis of a class of prohkms bevond the scope of any previous AI theory. Our analysis suggests that a qualitative physics for solid objects shoudd include the following features. among uthers:

- A rich geometrical theory, inctuding topological. metric, and differential descriptors. and speciaj shapes.

- An account of the behavior of physical systems wer extended intervals of time. Such in zecount should incorporate constraints placed bv une object on inother: conservation laws. especially conserviation of energy: the principle that i phycieal system tencs tow arids a itable resting point: and in account of the net effects of collisions over extended time perions.

- The ubility to determine the existenoc of a stabte coniiguration of objects within qualitutuvely described geometrical constraints.

- The sbility to calculate. exactly or qualitatively. important physical parameters suih is the enter of mass. [1+]

- The ability to bound the effect of small perturbations. On the negative side: We have not sbown that his ivpe of analysis is extensible to oover sil, or rosi. qualitative reasoning in this domain. We ave not stow $n$ that such an extension would be. in the long run. iny more parsimonious than simply enumerating special ases. is in the rule-based method rejected in section 2. We sase not ihown that any effective computationai methods an be developed on the basis of this theor: He cannot rive 1 inal resolution to these problems intil we ise impicmented a working ibstem. ind Jetermined the ringe of problems that it is adequate to address.

We plan to egin impiementution is de cloping - 7 sdequate geometric representation and inierence sistem. Citimately. we want to implement a physical reasonung system with all the features nentioned above.

\footnotetext{
- Hie reej the ground. because therwice ine ivpotheses i:e

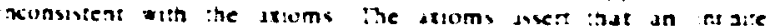

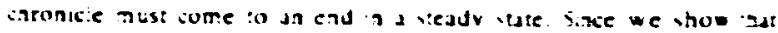
acie s wo vedur viate for the f:e a he iunacl. ae nust prov $x$ : atthe zround : a rest on
} 


\section{Acknowledgements}

Discussions with Sanjaya Addanki, Alex Botta, Pat Hayes, Leo Joskowie, and Drew McDermott have contributed immensely to the development of this theory. The research has been supported by NSF grants DCR8402309 and DCR-8603758.

11. References

(1) E. Davis, "A Logical Framework for Solid Object Physics," NYU Tech. Report \#245, 1986

[2] P. Hayes, The Naive Physics Manifesto", 1978: revised as "The Second Naive Physics Manifesto", in J. Hobbs and R. Moore, Formal Theories of the Commonsense World, ABLEX, 1985

[3] D. Brbrow, ed. Qualitative Reasoning about Physical Systems, MiT Press, 1985

[4] S. Fahlman, "A Planning System for Robot Construction Tasks," Arrificial Intelligence Journal. vol. 5. 1974. pp. 1-49

[5] J. de Kleer. "Qualitative and Quantitative Knowledge in Classical Mechanics," MIT AI Lab lech. Report $\$ 352,1975$

[6] A. Bundy, "Will it Reach the Top? Prediction in the Mechanics Worid, Arrificial Intelligence Jcurnal. Vol. 10. pp. 129-146

[7] K. Forbus. "A Study of Qualitative and Geometric Knowledge in Reasoning about Motion," MIT AI Lab Tech. Report $\$ 615$. 979

[8] B. Funt, "Problem Solving with Diagrammatic Representations," Artificial Intelligence Journal, vol. 13,1980 , p. $201-230$.

[9] G. Novak, Representations of Knowledge it. 3 Program for Solving Phvsics Problems," IJCAI 5. 1977 , pp. 286-291

[10] Y. Shoham. Najve Kinematics: One Aspect of Shape." IJCAV 9. 1985. pp. 436-142

[11] E. Davis, "Shape and Function of Solid Objects: Sorre Examples," VYU Tech. Repont $\neq 137$. 1984

[12] D.V. MeDermott. "A Temporal Logic Sor Reasoning about Plans and Processes." Cuqnanve Science. 1982 Fp. $101-155$

[1:] J. MoCarthy and P. Haves. "Some Philosopinical Proolems from the standooint of Artificial Intelligence. in Macnine intelligence, 3. Meltzer ind D. Michies (eds.), Edinburgn L'niversity Press. 1969

$[1+] A$. Bundy and $L$. Burd. "Tsing the Method of Fibres in Mecho to Culculate Rudii of Grration. in $D$. Gentner and $A$. Stevins. Meniul Hoiels. Erlbaum Associates, 1983 